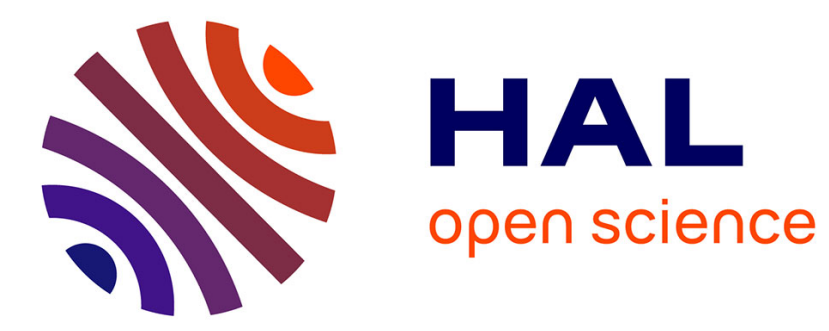

\title{
A particle filtering and kernel smoothing-based approach for new design component prognostics
}

Yang Hu, Piero Baraldi, Francesco Di Maio, Enrico Zio

\section{To cite this version:}

Yang Hu, Piero Baraldi, Francesco Di Maio, Enrico Zio. A particle filtering and kernel smoothingbased approach for new design component prognostics. Reliability Engineering and System Safety, 2015, 10.1016/j.ress.2014.10.003 . hal-01265661

\section{HAL Id: hal-01265661 \\ https://hal.science/hal-01265661}

Submitted on 1 Feb 2016

HAL is a multi-disciplinary open access archive for the deposit and dissemination of scientific research documents, whether they are published or not. The documents may come from teaching and research institutions in France or abroad, or from public or private research centers.
L'archive ouverte pluridisciplinaire HAL, est destinée au dépôt et à la diffusion de documents scientifiques de niveau recherche, publiés ou non, émanant des établissements d'enseignement et de recherche français ou étrangers, des laboratoires publics ou privés. 


\title{
A Particle Filtering and Kernel Smoothing-based Approach for New Design Component Prognostics
}

\author{
Yang $\mathrm{Hu}^{1}$, Piero Baraldi ${ }^{1}$, Francesco Di Maio ${ }^{1}$, Enrico Zio ${ }^{1,2, *}$ \\ ${ }^{1}$ Politecnico di Milano, Department of Energy, via Ponzio 34/3, Milan, 20133, Italy \\ ${ }^{2}$ Chair on Systems Science and the Energetic challenge, European Foundation for New Energy- \\ Electricite' de France, Ecole Centrale Paris and Supelec, Paris, France
}

\begin{abstract}
This work addresses the problem of predicting the Remaining Useful Life (RUL) of components for which a mathematical model describing the component degradation is available, but the values of the model parameters are not known and the observations of degradation trajectories in similar components are unavailable. The proposed approach solves this problem by using a Particle Filtering (PF) technique combined with a Kernel Smoothing (KS) method. This PF-KS method can simultaneously estimate the degradation state and the unknown parameters in the degradation model, while significantly overcoming the problem of particle impoverishment. Based on the updated degradation model (where the unknown parameters are replaced by the estimated ones), the RUL prediction is then performed by simulating future particles evolutions. A numerical application regarding prognostics for Lithium-ion batteries is considered. Various performance indicators measuring precision, accuracy, steadiness and risk of the obtained RUL predictions are computed. The obtained results show that the proposed PF-KS method can provide more satisfactory results than the traditional PF methods.
\end{abstract}

Key words: Prognostics; Remaining Useful Life; Parameter Estimation; Particle Filtering; Kernel Smoothing; Battery

\section{INTRODUCTION}

Prognostics is an engineering discipline which is mainly focused on the estimation of the future degradation path, remaining useful life and potential risk associated to an equipment [1]. Technical approaches of prognostics can be broadly categorized into data-driven and model-based [1-5]. Modelbased prognostic methods use mathematical representations of the equipment degradation evolution in order to predict the equipment Remaining Useful Life (RUL) [6-9]. They usually require the knowledge of the values of the model parameters, which are typically estimated considering the results of experimental tests or by observing the degradation behaviors of similar components. In practice, the whole model-based prognostics process is divided into an off-line and an on-line phase. During the offline phase, a mathematical model of the equipment degradation is built using the available physical knowledge on the involved degradation mechanisms and historical degradation data. Some statistical or artificial intelligence methods are, then, applied to the available historical data in order to estimate model parameters. For example, in [10] the authors used an adaptive-network-based fuzzy inference system and robust relevance vector machine to build the steelmaking process model which reduced the

*Corresponding author. Tel.: +3902 2399 6340; fax: +3902 23996309

E-mail address: enrico.zio@polimi.it 
effect of noise and outliers in the historical data; in [11], a combined relevance vector machine and exponential regression method was used to estimate the ball bearings degradation and predict its RUL based on real world vibration-based degradation data; in [12, 13], the authors used artificial neural networks combined with optimization algorithms to estimate the State-of-Charge of batteries. During the on-line phase, the acquired degradation measurements or condition monitoring data are used to adapt the degradation model to the current degradation situation and to predict the equipment RUL. For example, vibration data were used in [14] to predict the RUL distribution of bearings and the RUL distribution of Gyros were estimated using data collected during the testing process in [15].

The novelty of the present work is that we consider, within the framework of model-based prognostics, a case in which a mathematical model of the degradation process is available but the true model parameter values are unknown, and there are no data available for estimating them related to the degradation of similar components. These situations are typically encountered for safety-critical and high-value components which are characterized by very high reliability, unique or new design material composition. For these kinds of components, performing run-to-fail tests is too expensive or not feasible.

In such cases, the model parameters can be estimated by resorting to: i) expert knowledge, with uncertainty possibly expressed in the form of interval of values and ii) a sequence of data collected during the component operating life until the present time. In this setting of very limited available information, the objective is to jointly estimate i) the parameters of the degradation model ii) the remaining useful life of the component. For doing this, one has to handle the following sources of uncertainty i) measurement uncertainty, ii) intrinsic randomness of the degradation process, iii) uncertainty in the model parameters and iv) uncertainty on the future operational and environmental conditions.

In model-based prognostics, the problem of predicting the component RUL is typically tackled in two sequential steps: 1) estimation of the component degradation state at the present time and 2) prediction of the future evolution of the component degradation.

Step 1) is typically handled by resorting to filter methods such as Kalman Filter [16-18], used in cases of linear degradation models and Gaussian noise, and PF, used in cases of non-linear degradation models and/or non-Gaussian noise. Examples of applications of the PF methods to non-linear degradation problems can be found in [19-22]. Notice that all these works assumed that the model parameter exact values were known. In principle, also the uncertainty on the model parameters values can be treated by adopting Bayesian filtering methods where the dimension of the state vector is extended by including also the elements for the unknown parameters [23-25]. The problem of joint estimation of system state and model parameters had been addressed in [25, 26] considering Bayesian filtering approaches. For the application of fault detection, an approach to estimating the model parameters and the system state was discussed in [26]. In the context of prognostics, with few model parameters to be estimated, Bayesian approaches had been presented in [27-29]; however, these prognostic approaches had not been developed for cases with several model parameters and very poor knowledge on their prior distributions. In particular, it has been shown that in those cases in which the true parameter values are located in the tail of the prior distributions or the prior distributions are characterized by large variances with respect to the parameter typical ranges, most particles weights tend to very small values after the Bayesian updating. Under such situations, traditional PF methods will suffer a serious particle impoverishment [30]; thus, in order to get satisfactory estimates, one would need to use a very large number of particles, with the consequence of increasing computational efforts, and rendering this solution unfeasible in practical cases. 
Alternatively, the problem of particle impoverishment has been addressed by adding an artificial evolution to the particles in order to maintain their diversity [23, 31]. However, it has been shown that adding an artificial evolution causes an increase of the variance of the particles, which may obstacle the convergence of the population towards the true model parameter values [25]. Furthermore, the variance of the artificial noise is a further hyper-parameter to which the estimation results are very sensible, and which may be difficult to set in the context of the information available in the present work.

In this work, in order to overcome the problem of particle impoverishment in a case in which few available degradation measurements are available, we consider a method for parameter estimation based on a Kernel Smoothing technique. The method was proposed in [25] in a completely different problem context and is here extended to a prognostics problem. The main advantage of the method is that it can solve the problem of impoverishment without the side effects of variance increase (i.e. without adding extra artificial noise on particles).

Once the equipment degradation state at the present time and the model parameters have been estimated, it is necessary to predict the future evolution of the equipment degradation trajectory (step 2). Notice that this requires going beyond the traditional use of filtering methods, since it involves future time horizons in which no measurements are available for the Bayesian updating. This issue is addressed by adopting a procedure proposed in [32] based on the simulation of the future evolution of the particles describing the component degradation state at the present time.

The main contribution of this work consists in the proposal of a systematic method for on-line RUL prognostics of degrading equipment in the cases that 1) the true values of the parameters in the degradation model are unknown and/or affected by large uncertainties; 2) historical operation data of degradation of similar equipments are unavailable. From the methodological point of view, the novelty consists in the application of a kernel smoothing procedure to a prognostic problem which requires the prediction of the future evolution of the component degradation. The developed method for model parameters estimation and RUL prediction is applied to a numerical case study regarding the degradation of a Lithium-ion battery. Various performance indicators measuring precision, accuracy, steadiness and risk of the obtained RUL predictions are considered.

The remainder parts of the paper are organized as follows. Section 2 illustrates the problem statement. In Section 3, the PF approach to prognostics is briefly recalled, whereas in Section 4, we introduce the PFKS method for the joint estimations of the degradation state and the model parameters. Sections 5 and 6 describe the prediction of the future degradation evolution and the component RUL. Section 7 shows a numerical application of the proposed method to the prognostic of the RUL of a Lithium-ion battery. In Section 8 , we draw some conclusions and suggest potential future work.

\section{Problem Statement}

The objective of the work is to develop a prognostic method for RUL prediction of a degrading component, and related uncertainty. The following sources of information are considered available:

$>$ A degradation model for the mathematical representation of the evolution of the equipment degradation. The mathematical model is typically obtained from a physical understanding of the degradation mechanism. In this work, we assume that the physical model can be formulated as a first order Markov Process:

$$
x_{t}=g\left(x_{t-1}, \mathbf{p}_{t-1}, \gamma\right)
$$


where $g(x, \mathbf{p}, \gamma)$ is the recursive transition function, $x_{t}$ is an indicator of the equipment degradation state at time $t, \mathbf{p}$ is the vector of the model parameters, whose true values are unknown, $\gamma$ is the process noise which represents the degradation process uncertainty. Model parameters and process noise are assumed to be constant during the life of the component.

$>$ The measurement equation which links the degradation state $x$ and its measurements. It is typically represented by a possibly non-linear function $h$ :

$$
z_{t}=h\left(x_{t}, \sigma_{m}\right)
$$

where $z_{t}$ is the measurement of $x_{t}$ at time $t$ and $\sigma_{m}$ is the measurement noise.

$>$ Degradation measurements $z_{t}(t=1,2, \ldots, T)$, collected during the operating life of the component, until the present time $T$. For simplicity, we assume that measurements have been performed at regular intervals from $t=1$ (in arbitrary units).

$>$ A failure threshold defining the maximum acceptable degradation state: the equipment is considered failed when its degradation exceeds the failure threshold. In this work, we assume that the failure threshold value is known [33].

Notice that degradation measurements performed on identical or similar components are considered to be not available. On the contrary, we assume the availability of a prior estimate of the probability density functions (PDF) of the initial degradation state $p\left(x_{0}\right)$ and the model parameters $p\left(\mathbf{p}_{0}\right)$ based on expert judgment.

\section{THE PARTICLE FILTERING-BASED APPROACH TO PROGNOSTICS}

The PF-based approach to prognostics is typically divided into three steps (Figure 1, the solid boxes are inputs and the dotted boxes are outputs):

1) a filtering step for the estimation of the equipment degradation state at the present time, which is based on the use of the prior PDF of the model parameters and state provided by the experts, the degradation model and the measurements performed until the present time

2) a prediction step for the estimation of the future degradation evolution using the posterior PDF of the model parameters and state (output of step 1), and the updated degradation model (where the unknown parameters are replaced by the estimated ones)

3 ) the prediction of the equipment RUL which is based on the degradation state prediction (output of step 2) and the knowledge of the failure threshold.

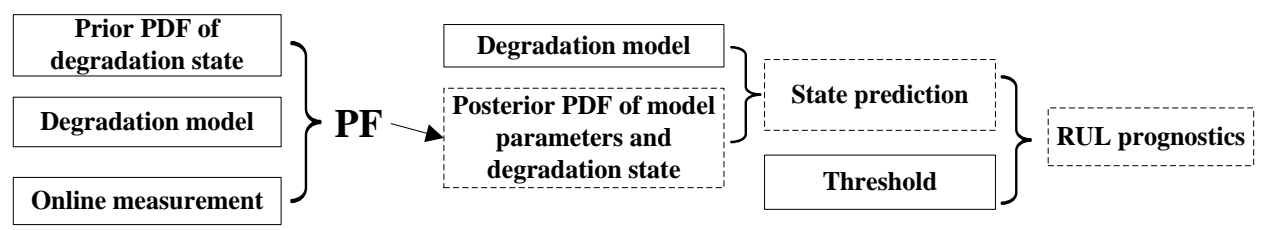

Figure 1 Sketch of the PF-based approach to RUL prognostics 
Section 4 will be devoted to the illustration of the proposed method for estimating the equipment degradation state and model parameters. Section 5 and 6 will present the methods for the prediction of the future degradation evolution and the estimation of component RUL, respectively.

\section{ESTIMATION OF THE COMPONENT DEGRADATION STATE AT THE PRESENT TIME AND OF THE MODEL PARAMETERS}

In order to facilitate the explanation of the method, in Section 4.1, we describe the basics of the PF estimation of the degradation state assuming that the parameters of the degradation model are known. Then, in Section 4.2 and 4.3, we discuss the problem of the joint estimations of the degradation state and the model parameters estimation. Section 4.4 describes the procedures of kernel smoothing.

\subsection{Particle filtering estimate of the degradation state}

In this filtering step, we use the degradation model, the noisy measurements and the prior PDF of the degradation state to estimate the posterior PDF of the degradation state. A detailed description of the PF method for the estimation of the system state can be found in $[8,34-36]$. The operative procedure is typically divided into two steps: prediction and update. In the prediction stage, one knows $p\left(x_{t-1} \mid z_{1: t-1}\right)$, and, by using Eq.(1) and the Chapman-Kolmogorov equation, the prediction distribution of the degradation at the next time $p_{f}\left(x_{t} \mid z_{1: t-1}\right)$ can be obtained:

$$
p_{f}\left(x_{t} \mid z_{1: t-1}\right)=\int p\left(x_{t-1} \mid z_{1: t-1}\right) p\left(x_{t} \mid x_{t-1}\right) d x_{t-1}
$$

When the new measurement $z_{t}$ arrives, one can update and calculate the posterior PDF $p\left(x_{t} \mid z_{1: t}\right)$ via the Bayesian rule:

$$
p\left(x_{t} \mid z_{1: t}\right)=\frac{p\left(x_{t} \mid z_{1: t-1}\right) p\left(z_{t} \mid x_{t}\right)}{\int p\left(x_{t} \mid z_{1: t-1}\right) p\left(z_{t} \mid x_{t}\right) d x_{t}}
$$

Usually, except for the situation of linear Gaussian state space models (Kalman filter) and hidden finitestate space Markov chains (Wohnam filter), it is not possible to evaluate analytically the PDF in Eq.(4), since this requires the calculation of complex high-dimensional integrals. The PF solution is, then, based on the Monte Carlo sampling method. By sampling a large number of samples (called particles) from a proposal PDF $q\left(x_{t} \mid z_{\mathrm{l}: t}\right)$, the estimated posterior PDF $p_{e}\left(x_{t} \mid z_{\mathrm{l}: t}\right)$ can be calculated as:

$$
p_{e}\left(x_{t} \mid z_{1: t}\right) \approx \sum_{i=1}^{N} w_{t}^{i} \delta\left(x_{t}^{i}-x_{t}\right)
$$

where $x_{t}^{i}(i=1,2, \ldots, N)$ are the particles sampled from $q\left(x_{t} \mid z_{1: t}\right)$ and $w_{t}^{i}$ is the weight associated to the particle $x_{t}^{i}$ given by:

$$
w_{t}^{\prime i}=\frac{p\left(z_{1: t} \mid x_{0: t}^{i}\right) p\left(x_{0: t}^{i}\right)}{q\left(x_{0: t}^{i} \mid z_{1: t}\right)} ; w_{t}^{i}=\frac{w_{t}^{\prime i}}{\sum_{i=1}^{N} w_{t}^{\prime i}}
$$


One of the most adopted choices is to consider the proposal PDF $q\left(x_{t} \mid z_{1: t}\right)$ as the transition function, namely $q\left(x_{t} \mid z_{1: t}\right)=p\left(x_{t} \mid x_{t-1}\right)$. In this way, using Eq.(7), one can obtain the particle weights $w_{t}^{i}$ at time $t$ :

$$
w_{t}^{\prime i}=w_{t-1}^{\prime i} p\left(z_{t} \mid x_{t}^{i}\right) ; w_{t}^{i}=\frac{w_{t}^{\prime i}}{\sum_{i=1}^{N} w_{t}^{\prime i}}
$$

where $p\left(z_{t} \mid x_{t}^{i}\right)$ is called the likelihood of measurement $z_{t}$ given the particle $x_{t}^{i}$, which can be derived from the measurement function in Eq.(2).

The following pseudocode reports the procedural steps for the estimate of the degradation state at the present time.

Sample $x_{0}^{i} \sim p\left(x_{0}\right)$, set initial weight $w_{0}^{i}=N^{-1}, i=1,2, \ldots, N$

$\mathrm{t}=1$;

While (t<T_end)

Sample $x_{t}^{i} \sim p_{f}\left(x_{t} \mid z_{0: t-1}\right)$;

Calculate the weight $w_{t}^{i}=w_{t-1}^{i} L\left(z_{t} \mid x_{t}^{i}\right) / \sum_{i=1}^{n} L\left(z_{t} \mid x_{t}^{i}\right)$

Calculate the state estimation and posterior PDF $\hat{x}_{t}=\sum_{i=1}^{N} w_{t}^{i} \times x_{t}^{i}, p_{e}\left(x_{t} \mid z_{1: t}\right) \approx \sum_{i=1}^{N} w_{t}^{i} \delta\left(x_{t}^{i}-x_{t}\right)$

Calculate the criteria for resampling $\operatorname{ESS}_{t} \approx\left(\sum_{i=1}^{n}\left(w_{t}^{i}\right)^{2}\right)^{-1}$

if $\operatorname{ESS}_{t} \leq N / 2$

resample with probability $\mathrm{P}\left(k_{t}^{i}=k_{t-1}^{i}\right)=w_{t-1}^{i}$

end if

$\mathrm{t}=\mathrm{t}+1$;

end while

\subsection{Combined State and Parameter estimation}

Several methods such as Maximum likelihood, Expectation Maximization, Minimum Mean Square Error and other hybrid methods have been used to estimate degradation model parameters on the basis of a batch of historical data [37-41]. The main limitation of the application of these approaches to prognostics is that they consider the problem of parameter estimation distinct from that of RUL prognostics. This causes that every time a new degradation measurement becomes available, a completely new estimation of the parameters needs to be performed. Then, with the new parameter values, the prognostic model has to be newly applied, without any benefits from the previous results.

Also Bayesian approaches such as the Overall Bayesian Method (OBM) and the Recursive Bayesian Method (RBM) were applied to the problem of parameter estimation in prognostic problems [42-44]. Although OBM provides satisfactory results, it cannot be used in this work since it relies on the use of historical batch data. In [44], the authors compared the performance of OBM, RBM and PF with respect 
to the RUL prognostic objective; the obtained results show that PF is the most suitable method for online parameter estimation and RUL prognostics.

\subsection{Combined State and Parameter estimation using PF}

The combined estimate of the equipment degradation state and model parameters can be performed by using an augmented PF [25, 34, 44-46]. The idea is to consider the model parameters as elements of the state vector which is estimated by the PF. Thus, the generic augmented $i$-th particle $k_{t}^{i}$, is represented by:

$$
k_{t}^{i}=\left\{x_{t}^{i}, \mathbf{p}_{t}^{i}, w_{t}^{i}\right\}
$$

where $x_{t}^{i}$ represents the degradation state, $\mathbf{p}_{t}^{i}$ the model parameters at time $t$ and $w_{t}^{i}$ is the weight associated to the particle. Eq.(1) becomes a system of two equations, one describing the transition of the state and the other the transition of the parameters:

$$
\begin{aligned}
x_{t}^{i} & =g_{1}\left(x_{t-1}^{i}, \mathbf{p}_{t-1}^{i}, \gamma_{1}\right) \\
\mathbf{p}_{t}^{i} & =g_{2}\left(\mathbf{p}_{t-1}^{i}, \gamma_{2}\right)
\end{aligned}
$$

Different strategies have been considered to set the function $g_{2}$ and, thus, to simulate the evolution of the model parameters values. In [44], the distributions of the parameters were kept unchanged during the prediction stage:

$$
p\left(\mathbf{p}_{t} \mid z_{1: t-1}\right)=p\left(\mathbf{p}_{t-1} \mid z_{1: t-1}\right)
$$

Thus, within a PF approach, the evolution of the values of the parameters in the particles becomes:

$$
\mathbf{p}_{t+1}^{i}=\mathbf{p}_{t}^{i}
$$

This approach will be here referred to as Particle Filtering with unchanged parameter distribution (PFUC). In [47], a dual PF method was used to handle the problem: the main idea of this dual PF approach is to decouple the state and parameters estimates and preform them sequentially: one PF is used for the degradation state estimate and the other PF for the model parameters estimate.

These two options have been shown to properly work when few model parameters are to be estimated, but they tend to suffer the problems of particle degeneracy and impoverishment in the case in which several model parameters needed to be estimated [48]. Resampling methods [34, 49], such as bootstrap resampling with replacement, can be used to avoid the problem of particle degeneracy, but are typically not effective with respect to the problem of particle impoverishment, since they tend to reduce the diversity in the particle population. Advanced resample methods such as those described in [50, 51] overcome the problem of particle impoverishment in low dimension of state space, but they have been shown to be not effective in high dimensional state space.

The problem of the particle impoverishment has been addressed in several works [23, 52, 53] by adding an artificial evolution to the particles parameters evolution equation. In practice, Eq.(10) becomes:

$$
\mathbf{p}_{t+1}^{i}=\mathbf{p}_{t}^{i}+N\left(0, \sigma_{A N}^{2}\right)
$$

where $\sigma_{A N}^{2}$ is the variance of the artificial noise. This approach will be referred to as Particle Filtering with Artificial Evolution (PF-AE). Although it has been shown that the method is able to provide more accurate estimates of the parameter values, it is difficult to apply it to our prognostic problem since it 
requires a proper setting of the value of the variance $\sigma_{A N}^{2}$, which is difficult to achieve by trial and error attempts, due to the unavailability of complete test trajectories. In [27, 52, 54, 55], the authors showed that, if too small values of $\sigma_{A N}^{2}$ are used, the particles convergence to the parameter true values is too slow and the problem of particle impoverishment was still be encountered. Whereas, if large values of $\sigma_{A N}^{2}$ are used, the convergence to the parameters true values was not achieved. Furthermore, it was shown that adding an artificial noise to the parameters values cause an increase of the variance of the particle population according to:

$$
V\left(\mathbf{p}_{t+1}^{i}\right)=V\left(\mathbf{p}_{t}^{i}\right)+\sigma_{A N}^{2}
$$

where $V\left(\mathbf{p}_{t+1}^{i}\right)$ is the variance of the parameters in the population after the artificial evolution is added [25]. In practice, every time we add the artificial evolution, the variance of the parameter value $\mathbf{p}_{t}^{i}$ will increase by $\sigma_{A N}^{2}$.

In order to overtake these difficulties, in this work we consider an alternative PF approach based on the Kernel Smoothing algorithm.

\subsection{The Kernel Smoothing algorithm}

Kernel smoothing is based on the application of two different procedures to the population of particles: shrinkage and perturbation. Shrinkage aims at moving the particle parameters values toward their estimated values, whereas perturbation adds a controlled noise to the particle parameters in order to maintain the desired variance in the population $[45,56,57]$.

$>$ Shrinkage

The particle shrinkage is performed by applying to the particle model parameters values the transform:

$$
\tilde{\mathbf{p}}_{t}^{i}=\mathbf{p}_{t}^{i} \sqrt{1-h^{2}}+\hat{\mathbf{p}}_{t}\left(1-\sqrt{1-h^{2}}\right)
$$

where the vector $\tilde{\mathbf{p}}_{t}^{i}$ contains the parameters values of the $i$-th particle after the shrinkage. The direction of shrinkage is the expected value of the parameter values $\hat{\mathbf{p}}_{t}$, at time $t$. The kernel parameter, $h \in[0,1]$, determines the degree of shrinkage: higher is its value, deeper is the shrinkage. If $h=1$, the model parameters completely shrink to the expected values $\hat{\mathbf{p}}_{t}$; whereas if $h=0$, no shrinkage is applied to the parameters. To set $h$, some authors suggested using the value $h=0.1$ [45], whereas other authors suggested optimizing the value of $h$ using historical data [56].

After shrinkage, the parameters variance in the population of particles will decrease from $V\left(\mathbf{p}_{t}^{i}\right)$ to $\left(1-h^{2}\right) V\left(\mathbf{p}_{t}^{i}\right)$. Then, the parameter vector $\tilde{\mathbf{p}}_{t}^{i}$ is used in Eq.(13) to predict the degradation state value $x_{t+1}^{i}$.

\section{$>$ Perturbation}

Perturbation is used to find the particle parameters values $\mathbf{p}_{t+1}^{i}$, at time $t+1$. In practice, an artificial evolution, i.e. a noise of variance $h^{2} V\left(\mathbf{p}_{t}^{i}\right)$, is added to the particle parameters values at time $t$ : 


$$
\mathbf{p}_{t+1}^{i}=\tilde{\mathbf{p}}_{t}^{i}+N\left(0, h^{2} V\left(\mathbf{p}_{t}^{i}\right)\right)
$$

Notice that the effect of adding the perturbation is to increase the variance of the parameters values in the population:

$$
V\left(\mathbf{p}_{t+1}^{i}\right)=V\left(\tilde{\mathbf{p}}_{t}^{i}\right)+h^{2} V\left(\mathbf{p}_{t}^{i}\right)=V\left(\mathbf{p}_{t}^{i}\right)
$$

Thus, different from the approach of Eq.(11) based on the addition of a Gaussian noise on the parameter values, the combined effect of the shrinkage and perturbation procedures can keep unmodified the variance of the parameters values in the population.

In the following, we will call this method as PF-KS.

\section{PREDICTION OF THE FUTURE DEGRADATION EVOLUTION}

Once the posterior PDF of the equipment degradation state and the model parameter values have been estimated, it is possible to predict the future evolution of the equipment degradation trajectory. Notice that this requires going beyond the traditional use of filtering methods, since it involves future time horizons in which no measurements are available for the Bayesian updating in Eq.(6) [31]. In practice, the estimated posterior PDF, $p_{e}\left(x_{t} \mid z_{1: t}\right)$, obtained at the previous step, should be propagated through Eq.(1) until the future time of interest $t+l$. This requires to compute [32]:

$$
p_{f}\left(x_{t+l} \mid z_{1: t}\right)=\int \ldots \int \prod_{j=t+1}^{t+l} p\left(x_{j} \mid x_{j-1}\right) p_{e}\left(x_{t} \mid z_{1: t}\right) \prod_{j=t}^{t+l-1} d x_{j}
$$

where $p_{f}\left(x_{t+l} \mid z_{1: t}\right)$ is the predicted PDF of degradation state at time $t+l$. However, the numerical evaluation of the integral in Eq.(16) is a computationally highly demanding task. A review of methods for the computation of $p_{f}\left(x_{t+l} \mid z_{1: t}\right)$ can be found in [54]. In this work, in order to reduce the computation demand, we follow the procedure proposed in [32] based on the assumption that the error generated by considering the particle weights constant from time $t$ to time $t+l$ is negligible with respect to other sources of error, such as those caused by model inaccuracy or by the assumptions made for setting process and measurement noise [58]. According to this procedure, the predictive PDF of the degradation state at time $t+l, p_{f}\left(x_{t+l} \mid z_{1: t}\right)$, is given by:

$$
p_{f}\left(x_{t+l} \mid z_{1: t}\right) \approx \sum_{i=1}^{N} w_{t}^{i} \delta\left(x_{t+l}-x_{t+l}^{i}\right)
$$

where the particle state $x_{t+1}^{i}$ is obtained by iteratively applying Eq.(1) to the state $x_{t}^{i}$.

\section{PREDICTION OF COMPONENT RUL}

Once the predicted degradation state PDF, $p\left(x_{t+l} \mid z_{1: t}\right)$, is available, the RUL can be found by calculating the earliest time at which the degradation state exceeds the failure threshold $x_{t h}$ [19]. In practice, the RUL associated to the $i$-th particle at the present time $t$ is given by:

$$
R U L_{t}^{i}=\left\{\left(T_{t}^{i}-1-t\right) \mid g\left(x_{T_{t}^{i}-1}, \mathbf{p}_{t}^{i}, \gamma\right)<x_{t h}, g\left(x_{T_{t}^{i}}, \mathbf{p}_{t}^{i}, \gamma\right) \geq x_{t h}\right\}
$$


where $T_{t}^{i}$ can be found by iteratively applying Eq.(1) to simulate the particles evolution. Thus, the predicted RUL distribution is given by: $[8,21,36]$

$$
p_{f}\left(R U L \mid z_{1: t}, x_{i}<x_{t h}\right) \approx \sum_{i=1}^{N} w_{t}^{i} \delta\left(R U L-R U L_{t}^{i}\right)
$$

The details and derivations of Eq.(18) and (19) can be found in [8, 21].

\section{NUMERICAL APPLiCATION}

In this section, we investigate the performance of the proposed method in an application of RUL prognostics of a Lithium-ion battery.

\subsection{Degradation Model}

A detailed description of the battery degradation mechanism can be found in [23, 59-61]. The quantity typically used to represent the battery degradation state is the battery capacity $q(t)$. According to the model proposed in $[23,59]$, the battery degradation is characterized by a first phase during which the battery capacity slowly decreases, followed by a second phase characterized by a fast decreasing process. These two phases can be described by a double exponential model:

$$
q(t)=p_{1} \cdot \exp \left(p_{2} \cdot t\right)+p_{3} \cdot \exp \left(p_{4} \cdot t\right)+N\left(0, \sigma_{p}^{2}\right)
$$

where $p_{1}, p_{2}, p_{3}$ and $p_{4}$ are the four model parameters $\left(p_{1}, p_{3}\right.$ determine the initial state and $p_{2}, p_{4}$ the degradation rate), $t$ is the number of charge/discharge cycles experienced by the battery and $\sigma_{p}^{2}$ is the process noise. Generally, $p_{1}$ and $p_{4}$ are positive, $p_{2}$ and $p_{3}$ are negative. Eq.(20) can be rewritten in a recursive form as:

$$
\begin{aligned}
& q_{1}(t)=q_{1}(t-1) \cdot \exp \left(p_{2}\right)+N\left(0, \sigma_{1}^{2}\right) \\
& q_{2}(t)=q_{2}(t-1) \cdot \exp \left(p_{4}\right)+N\left(0, \sigma_{2}^{2}\right)
\end{aligned}
$$

where $q_{1}(t)$ and $q_{2}(t)$ are two first-order independent Markov processes, with Gaussian process noises characterized by variance $\sigma_{1}^{2}$ and $\sigma_{2}^{2}$, respectively. Within this formulation, the present degradation state $q(t)$ can be obtained from $q_{1}(t)$ and $q_{2}(t)$ as:

$$
q(t)=p_{1} \cdot q_{1}(t)+p_{3} \cdot q_{2}(t)
$$

Eq.(22) constitutes the degradation model corresponding to Eq.(21), whereas Eq.(20) is introduced only to compute the degradation state but is not used within the PF approach. The measurement equation is:

$$
Q(t)=p_{1} \cdot q_{1}(t)+p_{3} \cdot q_{2}(t)+N\left(0, \sigma_{m}^{2}\right)
$$

where $Q(t)$ is the measurement at the $t$-th charge/discharge cycle and $\sigma_{m}^{2}$ is the measurement noise. The failure threshold of $q(t)$ is set according to expert knowledge. 


\subsection{Generation of the Measurement Data and of the Battery Failure Times}

The performance of the proposed prognostics method has been verified considering 100 simulated battery degradation trajectories. Each degradation trajectory is characterized by different values of the parameters $p_{1}, p_{2}, p_{3}$ and $p_{4}$, sampled from the statistical distributions reported in Table 1 . All the trajectories have been simulated considering the process and measurement noise and the failure threshold reported in Table 2.

Table 1 Distributions from which the true values of the parameters are sampled

\begin{tabular}{|c|c|c|c|c|}
\hline Parameter & $p_{1}$ & $p_{2}$ & $p_{3}$ & $p_{4}$ \\
\hline Value & $U(0.88,0.92)$ & $U(-0.0097,-0.0008)$ & $U(-0.0004,-0.0001)$ & $U(0.03,0.06)$ \\
\hline
\end{tabular}

Table 2 Process, measurement noise and threshold value

\begin{tabular}{|c|c|c|c|c|}
\hline Parameter & $\sigma_{1}$ & $\sigma_{2}$ & $\sigma_{m}$ & threshold \\
\hline Value & 0.0001 & 0.001 & 0.001 & 0.7172 \\
\hline
\end{tabular}

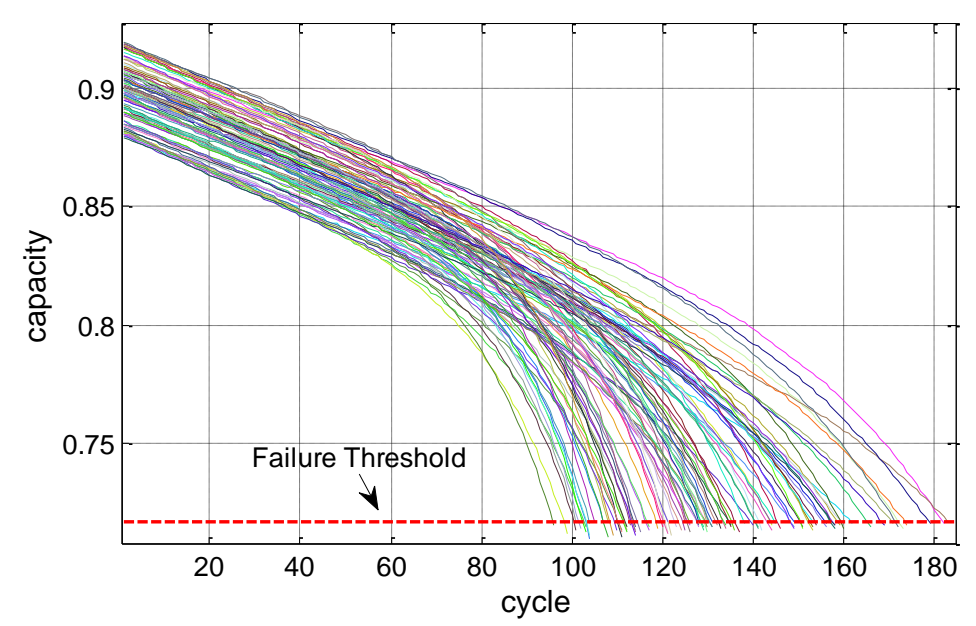

Figure 2100 batteries degradation trajectories

Figure 2 shows the 100 simulated degradation trajectories. Due to the different values of the parameters, life durations are uncertain, ranging from 95 to 182 cycles. The sampled values of $p_{1}, p_{2}, p_{3}$ and $p_{4}$, the obtained degradation state $q$ and the failure time will be referred to as the "true" values.

\subsection{Results}

The experiment is performed assuming that the true values of $p_{1}, p_{2}, p_{3}$ and $p_{4}$ are not known, and the measurements performed on the test battery, i.e. the batteries whose RUL we want to predict, are available from cycle 1 to the present cycle.

Notice that the application of the proposed method for the joint estimate of the component degradation state and model parameter values has an important prerequisite known as observability. Given that in case of non-observable systems the application of filtering-based method can produce poor estimates, one should verify whether the system state vector (degradation state and model parameters) is 
observable. Whereas for linear systems the global observability of the system state can be verified by computing the rank of the observability matrix, in non-linear systems the problem is more complex. According to [62], system observability is influenced by several factors such as the prior distributions of the degradation state and model parameters, and an adequate characterization of the noise sources. In the present work, the problem of discussing the observability of the system is not addressed, but, since we are considering an artificial numerical case study, we will discuss the accuracy of the obtained results considering also different possible choices of the prior distributions. Interested readers can find in [63], an example of demonstration of the observability of a non-linear system within a particle filtering problem.

\subsubsection{Parameters and Degradation State Estimation}

In this section, we analyze the parameters and degradation state estimation obtained by the PF-KS algorithm of one of the 100 simulated batteries. The prior PDFs and the true values of the parameters $p_{1}, p_{2}, p_{3}$ and $p_{4}$ are reported in Table 3 .

Table 3 The prior PDFs used for the four parameters

\begin{tabular}{|c|c|c|c|c|}
\hline Parameter & $p_{1}$ & $p_{2}$ & $p_{3}$ & $p_{4}$ \\
\hline Prior distribution & $U(0.85,1.2)$ & $U(-0.001,0)$ & $U(-0.001,0)$ & $U(0.03,0.13)$ \\
\hline True value & 0.887 & $-8.86 \mathrm{e}-4$ & $-2.32 \mathrm{e}-4$ & 0.0458 \\
\hline
\end{tabular}

Notice that the prior PDFs are remarkably more dispersed than those used for the simulation of the true values of these parameters (Table 1). Furthermore, the problem is complicated by the fact that the expected values of the true distributions of the four parameters are located in the tail of the prior distributions. This setting has been chosen in order to assess whether the method can work even if the parameters prior PDFs are very uncertain and their expected values are shifted with respect to the true values.

Figure 3 shows the estimates of the expected values and $90 \%$ confidence intervals of the four parameters. The continue thick horizontal lines represent the true value, the thin continuous lines the estimate of the parameters expected values and the dotted lines the $90 \%$ confidence interval of the parameters posterior distributions. Figure 4 shows the time evolution of the PDFs of parameters $p_{3}$ and $p_{4}$, with the thindotted line representing their true values. 

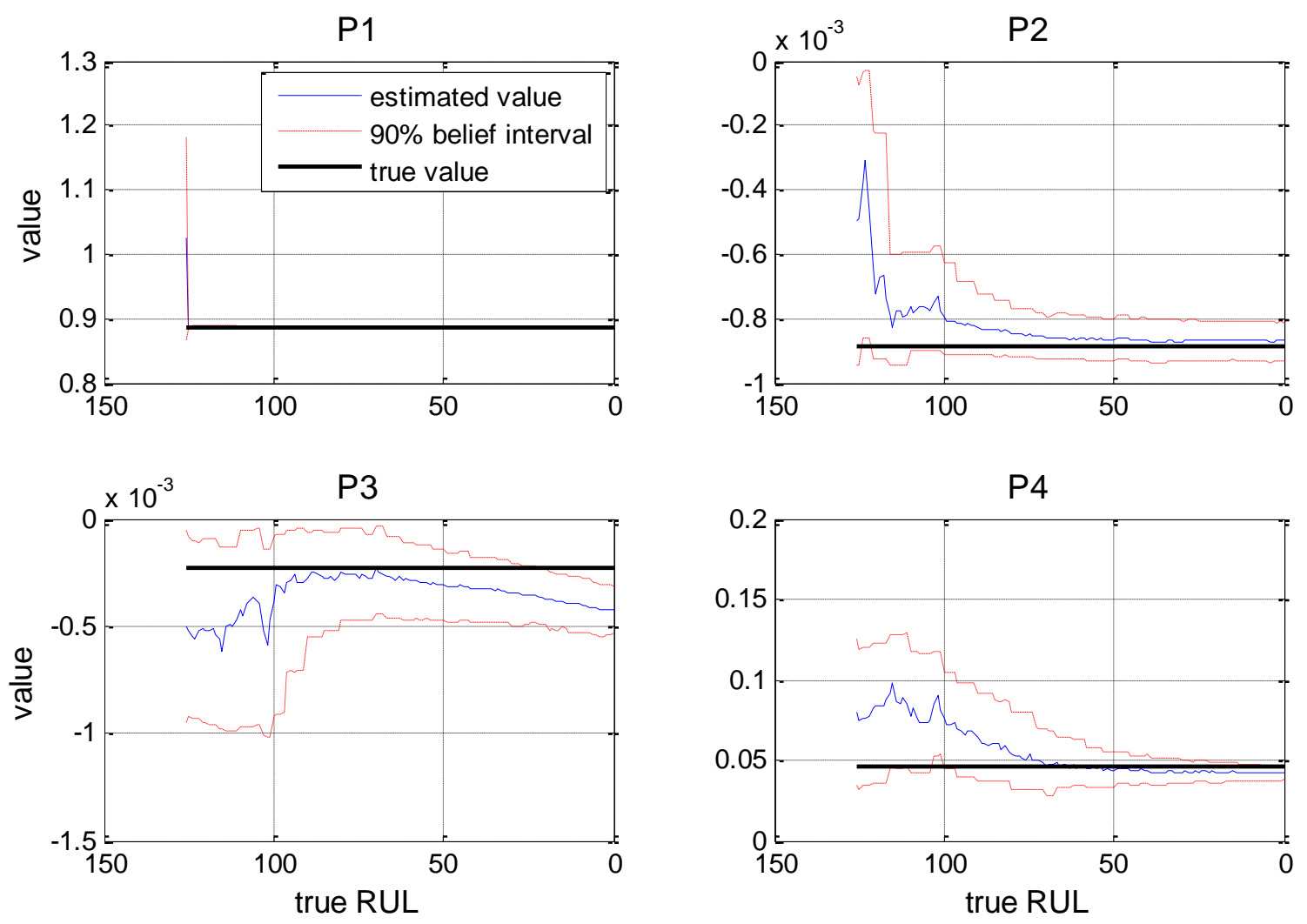

Figure 3 Parameters estimates

It can be observed that, as expected, the $90 \%$ confidence interval width tends to decrease as time passes. Furthermore, the error in the estimates of parameters $p_{1}$ and $p_{2}$ is very small, and on $p_{4}$ is still satisfactory, whereas the algorithm seems not able to find the correct value of $p_{3}$ and reduce the uncertainty on its estimate. In particular, during the last part of the battery life, the $p_{3}$ expected value remarkably drifts from the true $p_{3}$ value. 

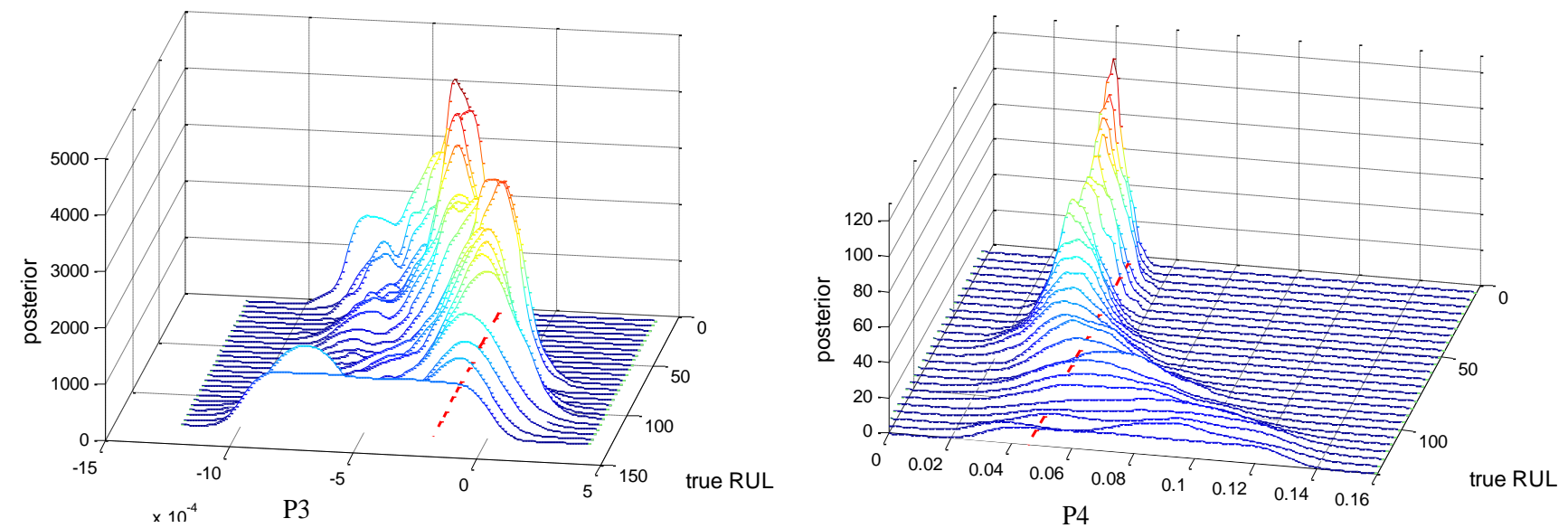

Figure 4 Posterior evolution of parameters $p_{3}$ and $p_{4}$

This high error on the $p_{3}$ estimate can be explained by considering the interaction between the estimates of the parameters. In particular, it seems that the error done in the estimate of $p_{3}$ tends to compensate the small error on the the estimate on $p_{4}$. In this respect, it should be noticed that if we repeat the estimation of $p_{1}, p_{2}, p_{3}$, assuming to know the correct value of $p_{4}$ (Figure 5), PF-KS is able to correcly estimate $p_{3}$. A possible explanation of this effect is that there can be different combinations of $p_{1}, p_{2}, p_{3}$ and $p_{4}$ values which can lead to satisfactory estimates of the current degradation state, but only one of them is satisfactory from the point of view of the model parameters. 

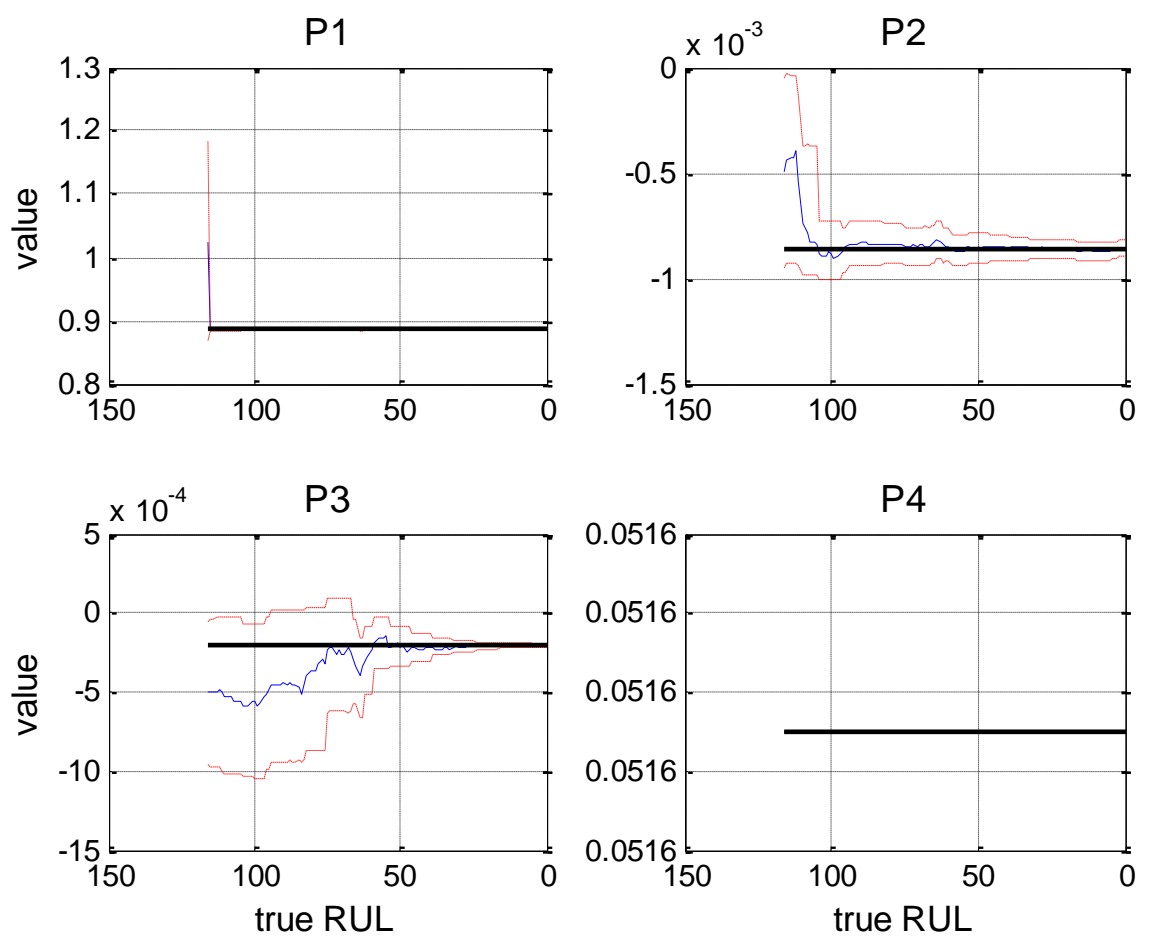

Figure 5 Parameters estimation given the true value of $p_{4}$

Figure 6 shows the absolute error in the prediction of the battery capacity degradation state and its $90 \%$ confidence interval. The estimate is very satisfactory since the error range is always below 0.002 .

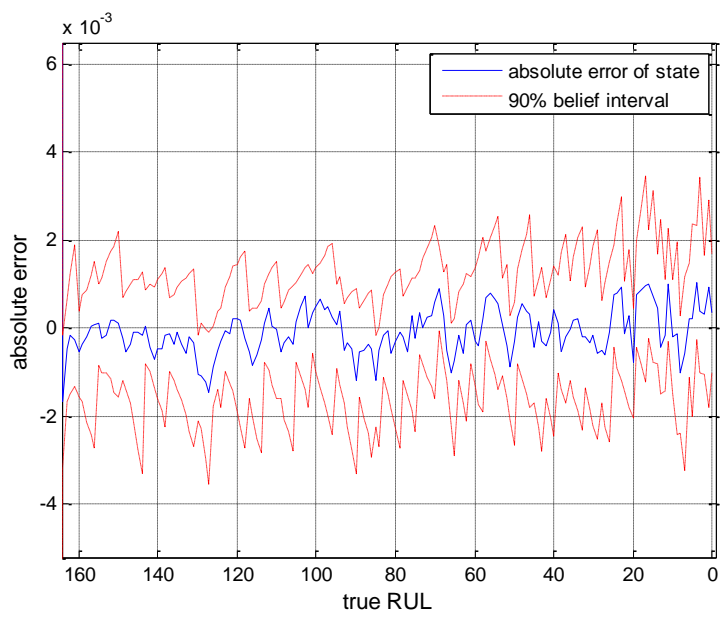

Figure 6 Absolute error on the degradation state 


\subsubsection{Prediction of the battery RUL}

In this section, we show the RUL prediction of the same battery analyzed in subsection 7.3.1. Figure 7 (left) shows the RUL predictions obtained at different times, with the thin-dotted line representing the 90\% confidence interval.
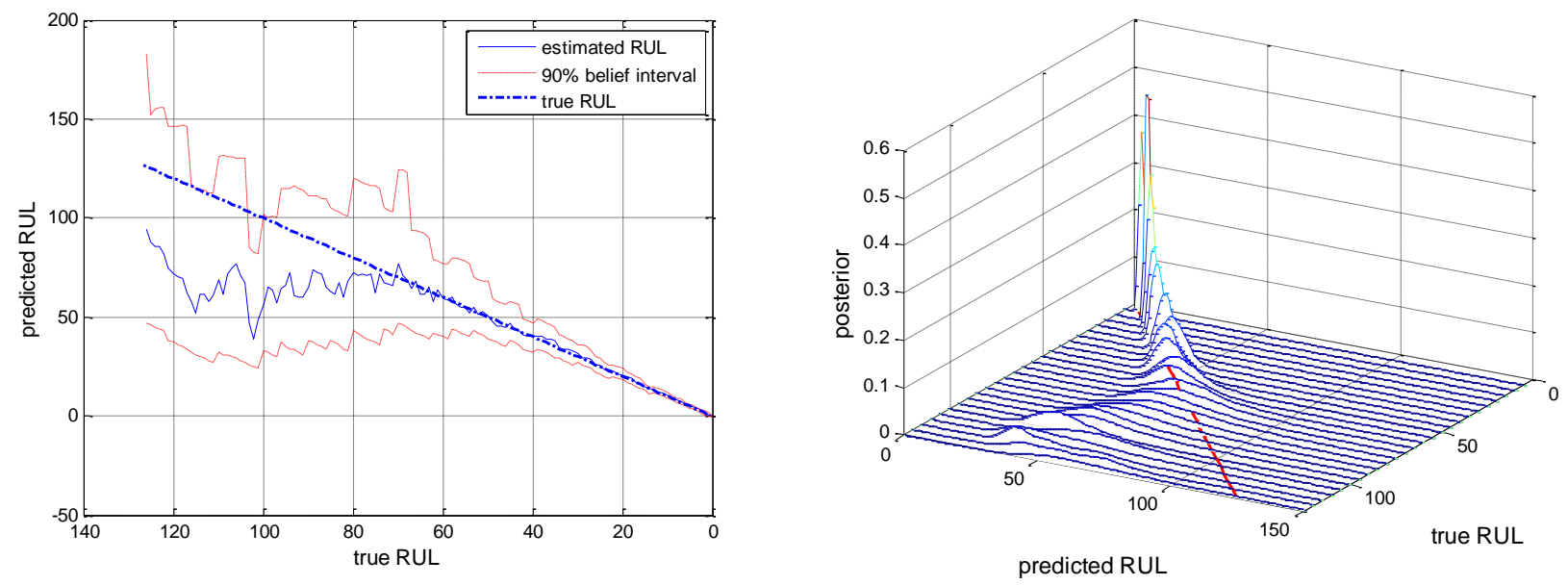

Figure 7 RUL prediction for the considered degradation trajectory

Figure 7 (right) shows the time evolution of the estimate of the RUL posterior distribution. Notice that before the true RUL reaches the value of 80 cycles, the RUL estimate tends to be very uncertain. This is due to the fact that at the beginning of the battery life, the degradation process is mainly influenced by the process $q_{1}$ whereas the overall contribution of $q_{2}$ is marginal (in Figure 8). Thus, the particle filtering algorithm is able to properly estimate parameters $p_{1}$ and $p_{2}$, which have a remarkable effect on the battery degradation state at the beginning of the battery life, whereas not effective with respect to parameters $p_{3}$ and $p_{4}$, which have negligible influence on the battery degradation state at the beginning. However, as time passes, the contribution of $q_{2}$ becomes predominant and, the PF-KS algorithm is able to improve the estimates of parameters $p_{3}$ and $p_{4}$, thus obtaining a good prediction of the RUL.

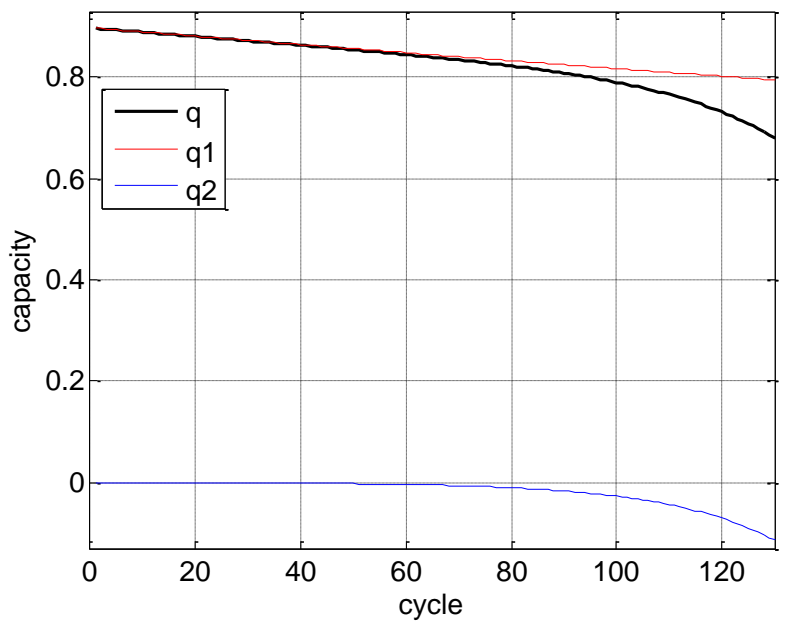

Figure 8. Effects of the terms $q_{1}$ and $q_{2}$ on the battery degradation $q$ 
In order to obtain a more robust evaluation of the performance metrics, the prediction of the RUL has been performed for all the 100 true degradation trajectories. Notice that the application of the method to a given battery is based only on the capacity measurements simulated for that trajectory and does not employ any information coming from the measurements of the other 99 trajectories.

We measure the performance of the different PF approaches, i.e. PF-UC, PF-AE and PF-KS from the point of view of the precision, accuracy, steadiness and risk level metrics described in [31, 64] and here briefly recalled:

$>$ Precision Index $(\mathrm{PI})$

PI considers the relative width of the prediction interval, which is defined by:

$$
P I_{t}=\frac{\sup \left(R U L_{-} I_{t}\right)-\inf \left(R U L_{-} I_{t}\right)}{R U L_{t}}
$$

where $\sup \left(R U L_{-} I_{t}\right)$ and $\inf \left(R U L_{-} I_{t}\right)$ are the upper and lower bounds of the RUL prediction $90 \%$ belief interval and $R U L_{t}$ is the real RUL at time $t$. Small values of $P I_{t}$ indicate more precise predictions.

Accuracy Index (AI)

$\mathrm{AI}$ is defined as the relative error of RUL prediction:

$$
A I_{t}=\frac{\left|R U L_{t}-R U L_{t}\right|}{R U L_{t}}
$$

where $R U L_{t}$ is the RUL estimated at time $t$. Small values of $A I_{t}$ indicate more accurate predictions.

Steadiness Index (SI)

SI measures the volatility of the RUL prediction:

$$
S I_{t}=\sqrt{\operatorname{var}\left(R U L_{(t-\Delta t): t}\right)}
$$

where $\Delta t$ is the sliding time window: in this paper we take $\Delta t=5$. Small values of $S I_{t}$ indicate more stable predictions.

$>$ Risk Index $(\mathrm{RI})$

RI is the probability of obtaining a RUL estimate smaller than the true RUL:

$$
R I_{t}=P\left(R U L \leq R U L_{t}\right)=\int_{-\infty}^{R U L_{t}} p\left(R U L_{-} I\right) d R U L
$$

where $p\left(R U L_{-} I\right)$ is the estimate of the RUL PDF. Large $R I_{t}$ values indicate conservative RUL prediction which are associated to lower risk for maintenance decisions.

In order to have a robust performance evaluation, we calculate the PI, AI, SI and RI indicators for the RUL predictions of the 100 batteries' degradation trajectories simulated in Section 7.2. Figure 9 reports the performance indicators averaged over the 100 trajectories, considering different values of the smoothing parameter $h$ in PF-KS. 

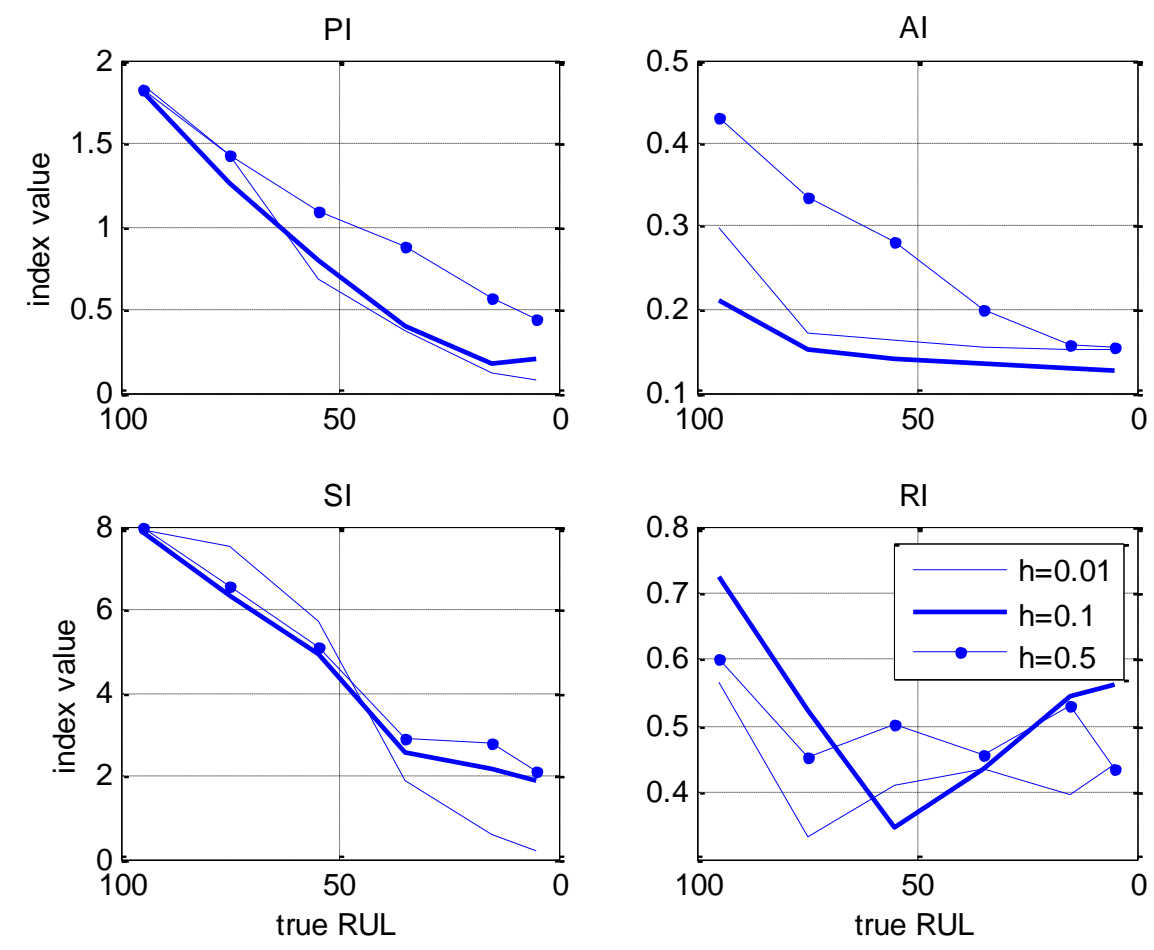

Figure 9 Performance comparison considering different values of the smoothing parameter $h$

At the beginning of the battery life, when the true RUL value is 95, the most satisfactory performance is obtained with $h=0.1$. As time passes and more data become available, $h=0.01$ leads to a faster convergence, as indicated by the lower values of the PI and SI indicators, although the RUL prediction is still less accurate (lower AI indicator) than that obtained using $h=0.1$. This effect is caused by the particle impoverishment problem that is faced when $h$ is too small. On the other side, larger values of $h$, such as 0.5 , do not allow a satisfactory convergence of the RUL and lead to unsatisfactory accuracy. Thus, our final choice has been to set the value of the parameter $h$ to 0.1 . Notice that the same value of $h=0.1$ is suggested in [45]. Current research work is devoted to the development of an algorithm for the automatic setting of the parameter, taking into account that its optimal value can change as time passes and that the $h$ value can be optimized each time a new measurement becomes available by considering the difference between the predicted and posterior PDFs.

Figure 10 reports the PI, AI, SI and RI indicators obtained by PF-UC, PF-AE and PF-KS. With respect to PF-AE, since there is no information or rule to set the variance of the artificial noise, we chose to use a noise variance equal to one percent of the variance of the parameter prior PDFs. This setting is supposed to provide enough variability in the particle model parameters without, at the same time, causing instabilities in the estimates. For PF-KS, the value of the smoothing parameter $h$ is equal to 0.1. For all three methods we have used the same number of particles $\mathrm{N}=10,000$, relatively large in order to not penalize the PF-UC in the comparison. 

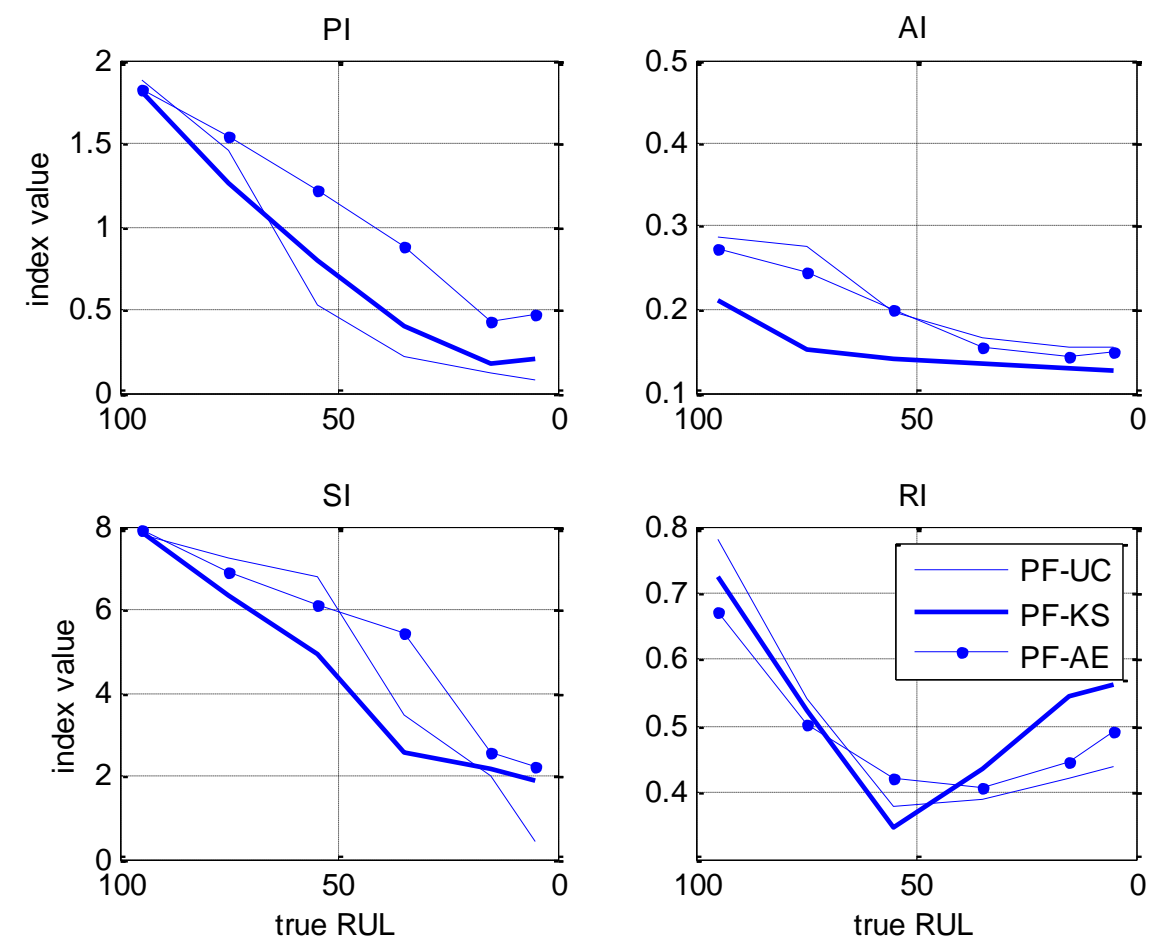

Figure 10 Performance comparison using PF-UC, PF-AE and PF-KS

Except for the precision indicator, PF-KS has the best performances. The lowest value of the PI indicator of PF-UC is due to the fact that at the end of the battery life all the particles are characterized by the same state and parameter values. Thus, $\sup \left(R U L_{-} I_{t}\right)$ and $\inf \left(R U L_{-} I_{t}\right)$ in Eq.(24) have very similar values, and PI becomes very small. Given the unsatisfactory accuracy of PF-UC (high value of $\mathrm{AI}$ ), we can conclude that this method overall performance is completely unsatisfactory.

Two analyses of the sensitivity of the PF-KS method with respect to the uncertainty in the prior distributions and the number of particles are performed.

Firstly, we have considered three possible situations of prior knowledge on the parameters values. The prior PDFs setting is shown in Table 4, where the intermediate level is the same as Table 3. Figure 11 shows the obtained results. Notice that although the quality of the prior PDFs has a remarkable influence on the overall performance at the beginning of the component life, the performances in the three cases become very similar as time passes and more measurements become available. This confirms that the PF-KS method is able to provide satisfactory RUL predictions even in case of limited knowledge on the parameter values.

Table 4 Three different parameter Prior Distributions settings

\begin{tabular}{|c|c|c|c|c|c|}
\hline \multicolumn{2}{|c|}{$\begin{array}{c}\text { very uncertain prior } \\
\text { distributions }\end{array}$} & \multicolumn{2}{c|}{$\begin{array}{r}\text { intermediate level of } \\
\text { uncertainty in the prior } \\
\text { distribution }\end{array}$} & \multicolumn{2}{c|}{$\begin{array}{c}\text { well defined prior } \\
\text { distributions }\end{array}$} \\
\hline$p_{1}$ & $p_{2}$ & $p_{1}$ & $p_{2}$ & $p_{1}$ & $p_{2}$ \\
$U(0.75,1.3)$ & $U(-1.5,0) \mathrm{e}-3$ & $U(0.85,1.2)$ & $U(-1,0) \mathrm{e}-3$ & $U(0.85,0.92)$ & $U(-9.7,-8) \mathrm{e}-4$ \\
\hline$p_{3}$ & $p_{4}$ & $p_{3}$ & $p_{4}$ & $p_{3}$ & $p_{4}$ \\
$U(-1.5,0) \mathrm{e}-3$ & $U(0.1,1.7) \mathrm{e}-1$ & $U(-1,0) \mathrm{e}-3$ & $U(0.3,1.3) \mathrm{e}-1$ & $U(-4,-1) \mathrm{e}-4$ & $U(0.3,0.6) \mathrm{e}-1$ \\
\hline
\end{tabular}



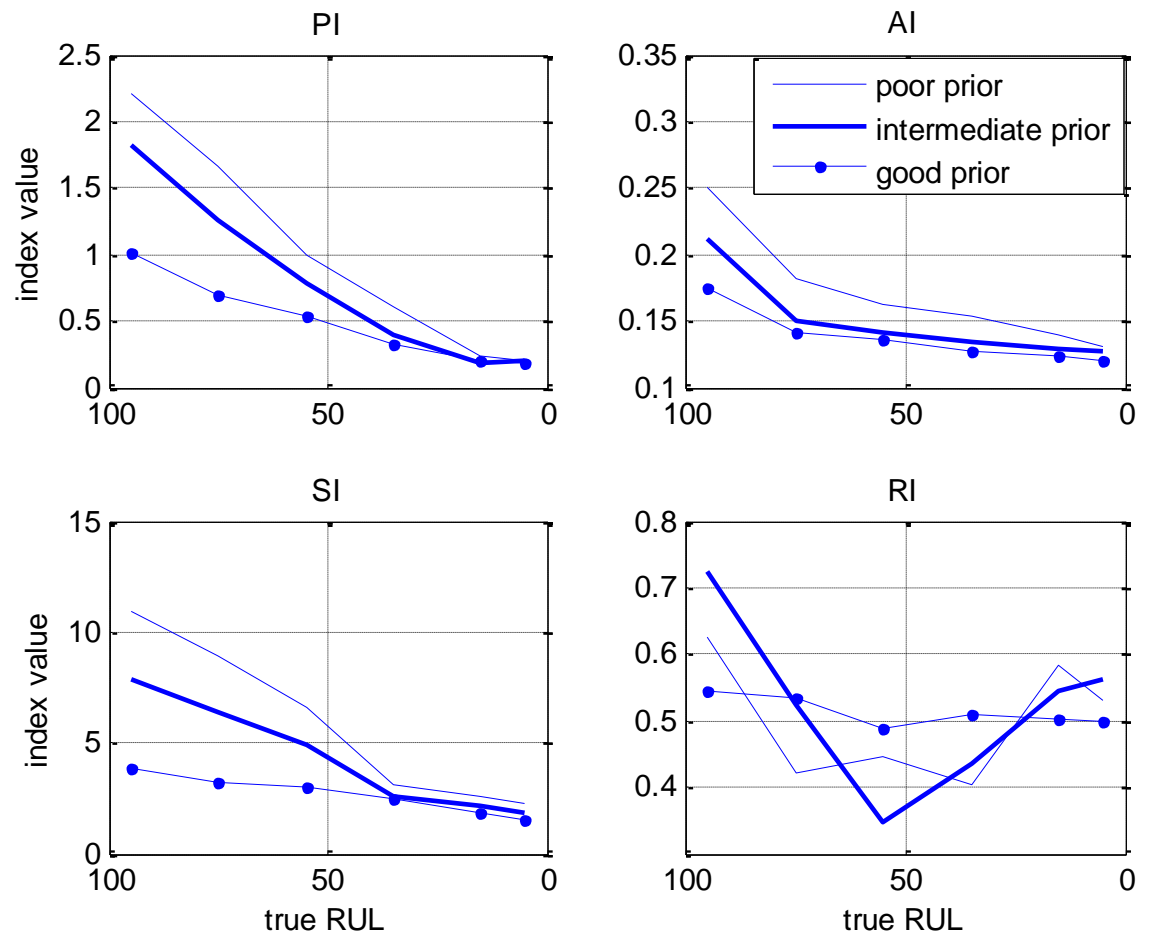

Figure 11 Comparison of the performance obtained using prior distributions of the model parameters characterized by different levels of uncertainty.
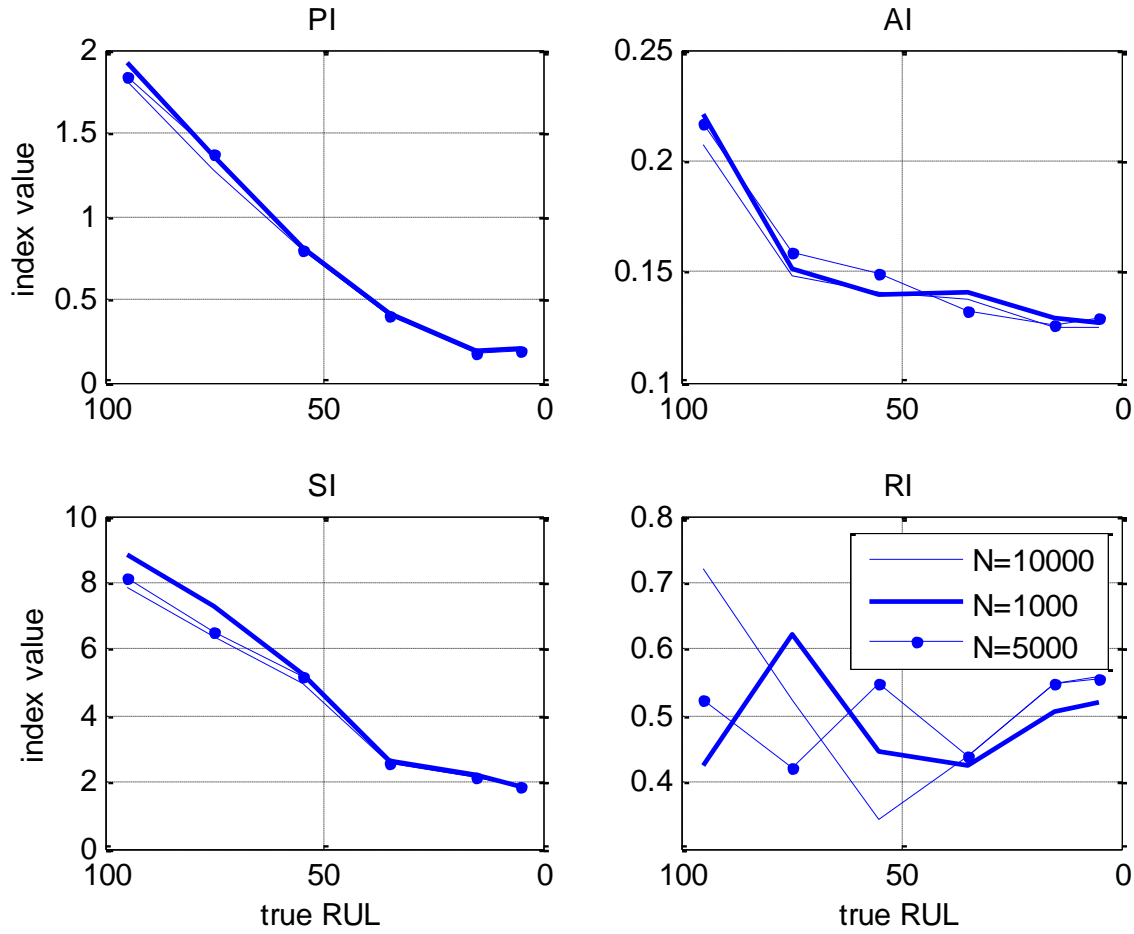

Figure 12 Performance comparison using three different numbers of particles 
Finally, Figure 12 shows the performances of the methods considering different numbers of particles $(1,000,5,000$ and 10,000). It can be observed that the performance indicators in the three cases are very similar. This confirms the ability of PF-KS of providing good performance without requiring high computational efforts.

\section{Conclusion}

In this work, we have proposed a particle filtering and kernel smoothing approach for the RUL prediction of degrading components based on a model of degradation with unknown parameters. We have assumed to know the model of degradation process and to be able to perform measurements of quantities related to the component degradation; on the other side, we have assumed that we do not know the true value of the degradation model parameters and the observations of degradation trajectories in similar components are unavailable.

The results obtained in a numerical case study regarding battery degradation have shown that the proposed method can provide estimates of the component RUL and of the model parameters which are more satisfactory than those obtained with other particle filtering approaches. From the point of view of the ability of the proposed approach to deal with the large uncertainties of the considered problem, at the beginning of the life of the component, when few observations of its degradation are available, the method provides, as expected, uncertain estimates of the model parameters and of the RUL, but as time passes and new information becomes available, the estimates become more precise and less uncertain.

The proposed approach will be further investigated in future works in order to understand if information on the sensitivity of the RUL to the different model parameters can be used to drive the search of their values more effectively. In particular, it is expected that by dynamically freezing the values of the less sensitive parameters, it is possible to improve the accuracy of the estimate of the most sensitive parameters. Furthermore, alternative methods for the prediction of the future component degradation trajectory, based on the estimate of the multidimensional integral in the Chapman-Kolmogorov equation with advanced Monte Carlo Techniques, will be explored.

\section{ACKNOWLEDGEMENTS}

Yang $\mathrm{Hu}$ gratefully acknowledges the financial support from China Scholarship Council (No. 201206110018). The participation of Enrico Zio to this research is partially supported by the China NSFC under grant number 71231001. The participation of Piero Baraldi and Francesco Di Maio is supported by the European Union Project INNovation through Human Factors in risk analysis and management (INNHF, www.innhf.eu) funded by the 7th framework program FP7-PEOPLE-2011- Initial Training Network: Marie-Curie Action.

The authors want to thank all the reviewers for their valuable comments to improve the quality of this paper.

\section{REFERENCES}

[1] Zio E. Prognostics and health management of industrial equipment. Diagnostics and Prognostics of Engineering Systems: Methods and Techniques. 2012:333-56.

[2] Si X-S, Wang W, Hu C-H, Zhou D-H. Remaining useful life estimation-A review on the statistical data driven approaches. European Journal of Operational Research. 2011;213:1-14.

[3] Sikorska J, Hodkiewicz M, Ma L. Prognostic modelling options for remaining useful life estimation by industry. Mechanical Systems and Signal Processing. 2011;25:1803-36. 
[4] Wang WB, Pecht M. Economic Analysis of Canary-Based Prognostics and Health Management. Ieee Transactions on Industrial Electronics. 2011;58:3077-89.

[5] Lee J, Wu F, Zhao W, Ghaffari M, Liao L, Siegel D. Prognostics and health management design for rotary machinery systems-Reviews, methodology and applications. Mechanical Systems and Signal Processing. 2013.

[6] Sankavaram C, Pattipati B, Kodali A, Pattipati K, Azam M, Kumar S, et al. Model-based and data-driven prognosis of automotive and electronic systems. Automation Science and Engineering, 2009 CASE 2009 IEEE International Conference on: IEEE; 2009. p. 96-101.

[7] Liu J, Wang W, Ma F, Yang Y, Yang C. A data-model-fusion prognostic framework for dynamic system state forecasting. Engineering Applications of Artificial Intelligence. 2012;25:814-23.

[8] Baraldi P, Cadini F, Mangili F, Zio E. Model-based and data-driven prognostics under different available information. Probabilistic Engineering Mechanics. 2013;32:66-79.

[9] Orchard ME, Hevia-Koch P, Zhang B, Tang L. Risk Measures for Particle-Filtering-Based State-of-Charge Prognosis in Lithium-Ion Batteries. Ieee Transactions on Industrial Electronics. 2013;60:5260-9.

[10] Han M, Zhao Y. Dynamic control model of BOF steelmaking process based on ANFIS and robust relevance vector machine. Expert Systems with Applications. 2011;38:14786-98.

[11] Di Maio F, Tsui KL, Zio E. Combining Relevance Vector Machines and exponential regression for bearing residual life estimation. Mechanical Systems and Signal Processing. 2012;31:405-27.

[12] Chang W-Y. Estimation of the state of charge for a LFP battery using a hybrid method that combines a RBF neural network, an OLS algorithm and AGA. Int J Elec Power. 2013;53:603-11.

[13] Eddahech A, Briat O, Bertrand N, Delétage J-Y, Vinassa J-M. Behavior and state-of-health monitoring of Li-ion batteries using impedance spectroscopy and recurrent neural networks. Int J Elec Power. 2012;42:487-94.

[14] Wang W. A model to predict the residual life of rolling element bearings given monitored condition information to date. IMA Journal of Management Mathematics. 2002;13:3-16.

[15] Wang Z, Hu C, Wang W, Zhou Z, Si X. A case study of remaining storage life prediction using stochastic filtering with the influence of condition monitoring. Reliability Engineering \& System Safety. 2014;132:186-95.

[16] Andre D, Nuhic A, Soczka-Guth T, Sauer DU. Comparative study of a structured neural network and an extended Kalman filter for state of health determination of lithium-ion batteries in hybrid electricvehicles. Engineering Applications of Artificial Intelligence. 2013;26:951-61.

[17] Sepasi S, Ghorbani R, Liaw BY. Improved extended Kalman filter for state of charge estimation of battery pack. Journal of Power Sources. 2014;255:368-76.

[18] Sepasi S, Ghorbani R, Liaw BY. A novel on-board state-of-charge estimation method for aged Li-ion batteries based on model adaptive extended Kalman filter. Journal of Power Sources. 2014;245:337-44.

[19] Baraldi P, Mangili F, Zio E. Investigation of uncertainty treatment capability of model-based and data-driven prognostic methods using simulated data. Reliability Engineering \& System Safety. 2013;112:94-108.

[20] Jouin M, Gouriveau R, Hissel D, Péra M-C, Zerhouni N. Prognostics of PEM fuel cell in a particle filtering framework. International Journal of Hydrogen Energy. 2014;39:481-94.

[21] Zio E, Peloni G. Particle filtering prognostic estimation of the remaining useful life of nonlinear components. Reliability Engineering \& System Safety. 2011;96:403-9.

[22] Miao Q, Xie L, Cui H, Liang W, Pecht M. Remaining useful life prediction of lithium-ion battery with unscented particle filter technique. Microelectronics Reliability. 2013;53:805-10.

[23] He W, Williard N, Osterman M, Pecht M. Prognostics of lithium-ion batteries based on Dempster-Shafer theory and the Bayesian Monte Carlo method. Journal of Power Sources. 2011;196:10314-21.

[24] An D, Choi J-H, Kim NH. Prognostics 101: A tutorial for particle filter-based prognostics algorithm using Matlab. Reliability Engineering \& System Safety. 2013;115:161-9.

[25] Tulsyan A, Huang B, Bhushan Gopaluni R, Fraser Forbes J. On simultaneous on-line state and parameter estimation in non-linear state-space models. Journal of Process Control. 2013;23:516-26.

[26] Bin Z, Sconyers C, Byington C, Patrick R, Orchard M, Vachtsevanos G. A Probabilistic Fault Detection Approach: Application to Bearing Fault Detection. Industrial Electronics, IEEE Transactions on. 2011;58:2011-8.

[27] Saha B, Goebel K. Model adaptation for prognostics in a particle filtering framework. International Journal of Prognostics and Health Management. 2011;2:10.

[28] Chen C, Pecht M. Prognostics of lithium-ion batteries using model-based and data-driven methods. Prognostics and System Health Management (PHM), 2012 IEEE Conference on: IEEE; 2012. p. 1-6.

[29] Sun J, Zuo H, Wang W, Pecht MG. Application of a state space modeling technique to system prognostics based on a health index for condition-based maintenance. Mechanical Systems and Signal Processing. 2012;28:585-96.

[30] Daum F. Nonlinear filters: beyond the Kalman filter. Aerospace and Electronic Systems Magazine, IEEE. 2005;20:5769. 
[31] Orchard M, Tobar F, Vachtsevanos G. Outer feedback correction loops in particle filtering-based prognostic algorithms: Statistical performance comparison. Stud Inform Control. 2009;18:295-304.

[32] Doucet A, Godsill S, Andrieu C. On sequential Monte Carlo sampling methods for Bayesian filtering. Stat Comput. 2000;10:197-208.

[33] Goebel K, Saha B, Saxena A. A comparison of three data-driven techniques for prognostics. 62nd Meeting of the Society For Machinery Failure Prevention Technology (MFPT)2008. p. 119-31.

[34] Arulampalam MS, Maskell S, Gordon N, Clapp T. A tutorial on particle filters for online nonlinear/non-Gaussian Bayesian tracking. Signal Processing, IEEE Transactions on. 2002;50:174-88.

[35] Cappé O, Godsill SJ, Moulines E. An overview of existing methods and recent advances in sequential Monte Carlo. Proceedings of the IEEE. 2007;95:899-924.

[36] Orchard ME, Vachtsevanos GJ. A particle-filtering approach for on-line fault diagnosis and failure prognosis. Transactions of the Institute of Measurement and Control. 2009;31:221-46.

[37] Chen B, Zhu Y, Hu J, Principe JC. 3 - Information Theoretic Parameter Estimation. In: Badong C, Yu Z, Jinchun H, Jose C. PrincipeA2 - Badong Chen YZJH, Jose CP, editors. System Parameter Identification. Oxford: Elsevier; 2013. p. 29-60.

[38] Wicker N, Muller J, Kalathur RKR, Poch O. A maximum likelihood approximation method for Dirichlet's parameter estimation. Computational Statistics \& Data Analysis. 2008;52:1315-22.

[39] Zhao Z, Huang B, Liu F. EM Algorithm for Parameter Estimation in Batch Process. In: Iftekhar AK, Rajagopalan S, editors. Computer Aided Chemical Engineering: Elsevier; 2012. p. 935-9.

[40] Eddahech A, Ayadi M, Briat O, Vinassa J-M. Online parameter identification for real-time supercapacitor performance estimation in automotive applications. Int J Elec Power. 2013;51:162-7.

[41] Yang Y, Liao Y, Meng G, Lee J. A hybrid feature selection scheme for unsupervised learning and its application in bearing fault diagnosis. Expert Systems with Applications. 2011;38:11311-20.

[42] An D, Choi J-H, Kim NH. Identification of correlated damage parameters under noise and bias using Bayesian inference. Structural Health Monitoring. 2012;11:293-303.

[43] Park B-J, Zhang Y, Lord D. Bayesian mixture modeling approach to account for heterogeneity in speed data. Transportation research part B: methodological. 2010;44:662-73.

[44] An D, Choi J-H, Kim NH. A Comparison Study of Methods for Parameter Estimation in the Physics-based Prognostics. 53rd AIAA/ASME/ASCE/AHS/ASC Structures, Structural Dynamics and Materials Conference; 2012.

[45] Liu J, West M. Combined parameter and state estimation in simulation-based filtering: Springer; 2001.

[46] Ching J, Beck JL, Porter KA. Bayesian state and parameter estimation of uncertain dynamical systems. Probabilistic Engineering Mechanics. 2006;21:81-96.

[47] Olivier LE, Huang B, Craig IK. Dual particle filters for state and parameter estimation with application to a run-of-mine ore mill. Journal of Process Control. 2012;22:710-7.

[48] Li T, Sun S, Sattar TP, Corchado JM. Fighting against Sample Degeneracy and Impoverishment in Particle Filters: Particularly on Intelligent Choices. arXiv preprint arXiv:13082443. 2013.

[49] Shi S, Chen D. Enhancing particle image tracking performance with a sequential Monte Carlo method: The bootstrap filter. Flow Measurement and Instrumentation. 2011;22:190-200.

[50] Li T, Sattar TP, Sun S. Deterministic resampling: Unbiased sampling to avoid sample impoverishment in particle filters. Signal Process. 2012;92:1637-45.

[51] Petetin Y, Desbouvries F. Optimal SIR algorithm vs. fully adapted auxiliary particle filter: a non asymptotic analysis. Stat Comput. 2013;23:759-75.

[52] Corbetta M, Sbarufatti C, Manes A, Giglio M. Stochastic Definition of State Space Equation for Particle Filtering Algorithms. Prognostic and System Health Management Conference, Milan, Italy2013.

[53] Higuchi T. Monte Carlo filter using the genetic algorithm operators. Journal of Statistical Computation and Simulation. 1997;59:1-23.

[54] Orchard M, Kacprzynski G, Goebel K, Saha B, Vachtsevanos G. Advances in uncertainty representation and management for particle filtering applied to prognostics. Prognostics and Health Management, 2008 PHM 2008 International Conference on: IEEE; 2008. p. 1-6.

[55] Daigle MJ, Goebel K. Model-Based Prognostics With Concurrent Damage Progression Processes. Ieee T Syst Man Cy-S. 2013;43:535-46.

[56] Chen T, Morris J, Martin E. Particle filters for state and parameter estimation in batch processes. Journal of Process Control. 2005;15:665-73.

[57] Wan-ping W, Sheng L, Ting-wen X. Particle filter for state and parameter estimation in passive ranging. Intelligent Computing and Intelligent Systems, 2009 ICIS 2009 IEEE International Conference on: IEEE; 2009. p. 257-61.

[58] Orchard ME. A particle filtering-based framework for on-line fault diagnosis and failure prognosis. 2007. 
[59] Saha B, Goebel K, Poll S, Christophersen J. Prognostics methods for battery health monitoring using a Bayesian framework. Instrumentation and Measurement, IEEE Transactions on. 2009;58:291-6.

[60] Zhang J, Lee J. A review on prognostics and health monitoring of Li-ion battery. Journal of Power Sources. 2011;196:6007-14.

[61] Marcicki J, Todeschini F, Onori S, Canova M. Nonlinear parameter estimation for capacity fade in Lithium-ion cells based on a reduced-order electrochemical model. American Control Conference (ACC), 2012: IEEE; 2012. p. 572-7.

[62] Mundnich Batic KB. Early detection of high volatility clusters using particle filters: Universidad de Chile; 2013.

[63] Mirabdollah MH, Mertsching B. Bearing only mobile robots' localization: Observability and formulation using SIS particle filters. Communications, Computing and Control Applications (CCCA), 2011 International Conference on: IEEE; 2011. p. 1-5.

[64] Olivares BE, Munoz C, Orchard ME, Silva JF. Particle-filtering-based prognosis framework for energy storage devices with a statistical characterization of state-of-health regeneration phenomena. Instrumentation and Measurement, IEEE Transactions on. 2013;62:364-76. 